\title{
Vital Imaging of Glomeruli in the Mouse Olfactory Bulb
}

\author{
Anthony-Samuel LaMantia, Scott L. Pomeroy, a and Dale Purves \\ Department of Neurobiology, Duke University Medical Center, Durham, North Carolina 27710 and Departments of \\ Anatomy and Neurobiology, Washington University School of Medicine, St. Louis, Missouri 63110
}

\begin{abstract}
We have monitored the pattern of identified glomeruli in the olfactory bulbs of newborn, juvenile, and adult mice over intervals of several hours to several weeks. Our purpose was to assess the development and stability of these complex units in the mammalian brain. Glomeruli can be observed by vital fluorescent staining and laser-scanning confocal microscopy without causing acute or long-term damage to brain tissue. Repeated observation of bulbs in the same animals between birth and 3 weeks of age showed that this region of the brain develops by progressive addition of these units to the original population. This increment occurs by the genesis of smaller new glomeruli between larger existing ones; no elimination of glomeruli was observed during this process. Finally, no addition (or loss) of glomeruli occurred in adult animals over a 2 week interval; once established, the number, size, and pattern of glomeruli are evidently stable.
\end{abstract}

The rules of circuit development and maintenance in the mammalian brain have usually been inferred from studies of fixed tissue. A more direct approach is to observe identified circuits in the same brain on more than one occasion. Recent advances in optical microscopy (White et al., 1987; Fine et al., 1988), digital image processing (Inoué, 1986), and the use of nontoxic vital fluorescent dyes (Purves et al., 1986; Magrassi et al., 1987) have enabled direct in vivo observation of neurons and their synaptic connections in the mammalian PNS for up to 6 months (Purves and Voyvodic, 1987). Here we describe the adaptation of these techniques to study circuit development and maintenance in the olfactory bulb of the mouse. We have chosen the olfactory system because iterated neural circuits called glomeruli are evident in the superficial layers of the bulb (Golgi, 1874). The distinctive morphology of glomeruli, their location near the surface of the brain, and their role as the initial processing units in the central olfactory pathway (for review, see Shepherd, 1972; Scott and Harrison, 1987) all recommend this class of circuits for in vivo analysis.

\footnotetext{
Received July 19, 1991 ; revised Oct. 4, 1991; accepted Oct. 18, 1991.

We thank Pat Newton, Dot Dill, and Ladan Hedayati for excellent technical assistance during all phases of this project and Mary Beth Groelle for photographic assistance. Several RH dyes were kindly furnished by Amiram Grinvald. The help of Chris Ho, David Simon, and Lewis Harris in some phases of this project is also gratefully acknowledged.

Correspondence should be addressed to Anthony-Samuel LaMantia, Ph.D., Box 3209, Department of Neurobiology, Duke University Medical Center, Durham, NC 27710 .

- Present address: Department of Neurology, Children's Hospital, Boston, MA 02115.

Copyright $(1992$ Society for Neuroscience $0270-6474 / 92 / 120976-13 \$ 05.00 / 0$
}

Assessing the development of complex neural units such as glomeruli is of interest for several reasons. Such iterated neural circuits (often called modules) have come to occupy a central position in current thinking about brain function, organization, and development (for reviews, see Mountcastle, 1978; Hubel, 1988; Rakic, 1988). Although a great deal is known about the development and regulation of the final numbers and arrangement of individual neurons, neuronal processes, and synapses, little information is available about the development of entire processing units, particularly those like glomeruli that occur in repeated patterns in various parts of the brain.

Accordingly, we have monitored changes in the size, shape, number, and distribution of glomeruli on the dorsal surface of the olfactory bulb in juvenile and adult mice. Such observations provide otherwise unobtainable information about the life history of complex circuits in the mammalian CNS. Glomeruli are added progressively in early life but remain as a stable population in adult animals.

Some of these results have been presented briefly (LaMantia and Purves, 1989).

\section{Materials and Methods}

Animals. Male albino mice (CF-1 strain; Harlan/Sprague-Dawley Labs, Indianapolis, IN) were used for this study. For neonatal studies, pups weighing $2.5-3.5 \mathrm{gm}$ were obtained at 3-5 d of age from litters born in our breeding colony. Female mice were housed separately after pairing for $24 \mathrm{hr}$ with a mature male; pregnant mothers were checked twice daily from postbreeding day 19 to reinsure that the day of birth was accurately recorded. On the third postnatal day, the mother and pups were removed from the colony and brought to the lab. After imaging experiments were completed and it was clear that the pups were not being rejected by their mother, the litter was returned to the colony. For studies in adult animals, male mice (25-30 gm, 8-12 weeks old) were obtained either from the colony or directly from the animal breeder.

Anesthesia and surgical procedures. Neonatal mice were rapidly anesthetized by cooling (Green, 1979). To maintain deep anesthesia, the pups were placed on a chilled brass plate fitted to the stage of a modified epifluorescence microscope. The plate was kept cold for the duration of the procedure (about $20 \mathrm{~min}$ ) by a small ice pack.

The skin was cleaned, incised, and held back by fine wire retractors anchored to the stage by magnets (Fig. $1 A$ ). The surgical field was bathed by a constant flow of sterile oxygenated Ringer's solution (Abbott Labs) at room temperature throughout the experiment. The bone over the right olfactory bulb was removed using a small electrical drill fitted with a fine dental bit. When the skull had been thinned to transparency, the bone flap was gently lifted away with jeweler's forceps to expose the dorsal surface of the olfactory bulb (Fig. $1 B$ ). Care was taken not to tear the underlying meninges; if the dura was damaged at this or any stage, the procedure was discontinued.

Adult mice were anesthetized with intraperitoneal acepromazine (25 $\mathrm{mg} / \mathrm{kg}$ ) and pentobarbital $(35 \mathrm{mg} / \mathrm{kg})$; a subcutaneous injection of $\mathrm{xy}-$ locaine $(0.5 \%)$ in the scalp was also made to provide additional local anesthesia. Once the animal was well anesthetized, the head was shaved and the trachea intubated with $1 \mathrm{~mm}$ o.d. polyethylene tubing. The mice 


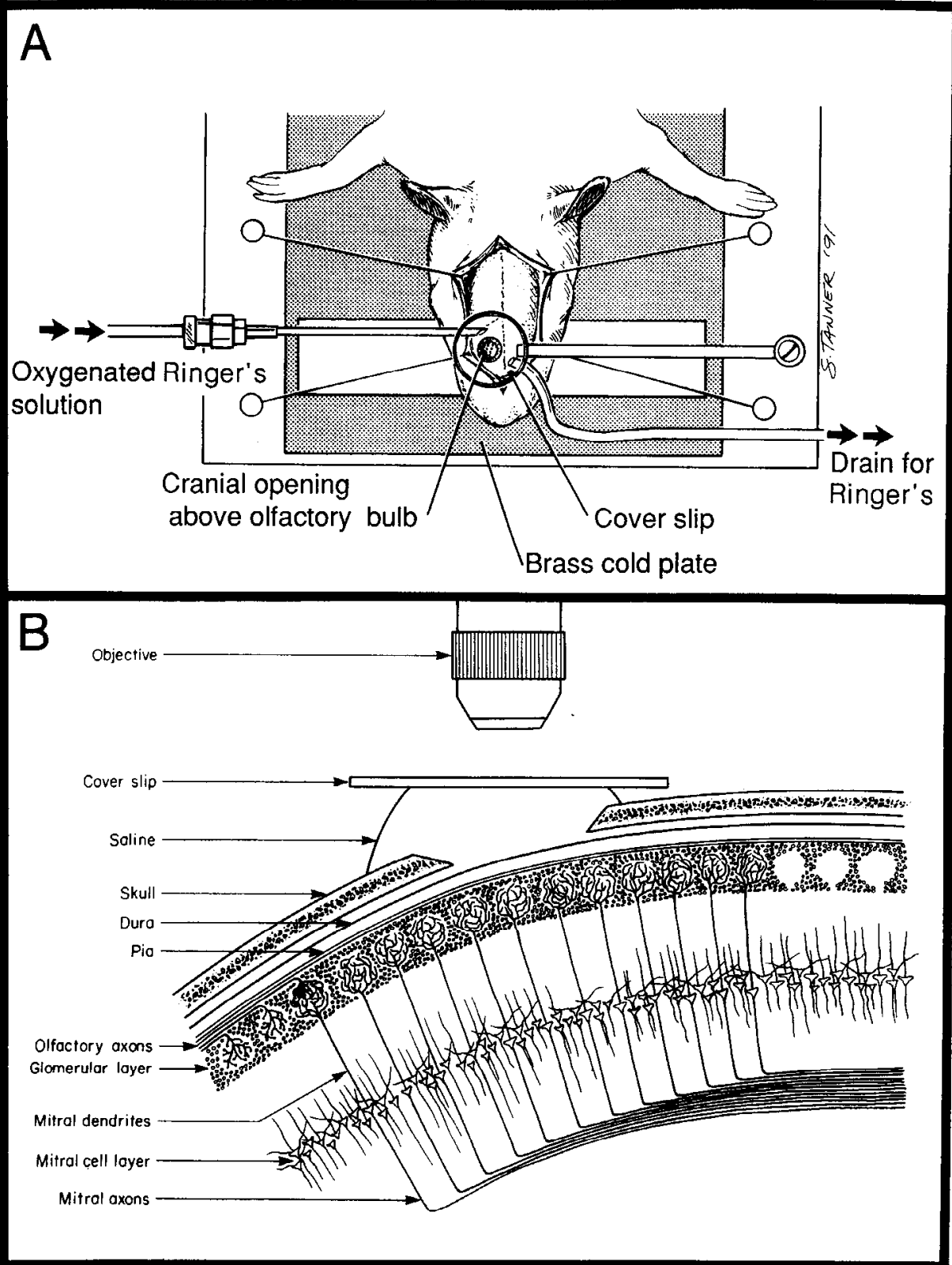

Figure 1. In vivo imaging of the mouse olfactory bulb. $A$, A mouse pup (or in some experiments an adult mouse) is placed upon a chilled brass plate. A small rod supports chin to level the anterior skull; in this configuration the dorsal surface of the bulb is parallel to the image plane of the microscope. A circle of bone $1-1.5 \mathrm{~mm}$ in diameter is then removed to expose the dura overlying the bulb. $B$, The relative positions of the dura, arachnoid, pia, nerve fiber layer, and glomerular layer are indicated in a more detailed schematic of the mouse olfactory bulb prepared for vital imaging. To stain glomeruli, the exposed dorsal surface of the bulb, still covered by the dura, is briefly exposed to the vital fluorescent dye RH414. In vivo images of the glomerular layer were acquired with a laser-scanning confocal microscope to reduce out-of-focus fluorescence arising from the overlying meninges and afferent fibers.

were ventilated with a mixture of moist $\mathrm{O}_{2}$ and room air for the duration of the procedure (120-140 cycles/min); body temperature was maintained with a heating pad. The surgical exposure of the olfactory bulb was similar to that for baby mice, except that a larger drill bit was used to remove the overlying bone.

Vital staining. Once exposed, the dorsal surface of the bulb was flushed with Ringer's solution and cleaned of connective tissue with a small wire loop. The superfusion was then interrupted and the meningeal surface exposed to a drop of $1.5 \mathrm{M} \mathrm{NaCl}$ for $3 \mathrm{~min}$ to breach the diffusion barrier presented by the dura, arachnoid, and pia (Fenstermacher and Rappoport, 1984). The hypertonic saline was removed by gentle suction and immediately replaced by a drop of a $2.5 \mathrm{~mm}$ solution of the styryl dye RH414 (Grinvald et al., 1984; obtained from Molecular Probes Inc., Eugene, $O R$ ). The dye solution was left in place for $4 \mathrm{~min}$ and then washed out by ongoing superfusion with Ringer's solution.

After washing for 3-5 min, a coverslip affixed to a spring-loaded magnetic base was placed over the surgical field (Fig. 1). The coverslip was maintained above the actual surface of the bulb, which was protected at all times by a layer of flowing Ringer's solution. The bulb was viewed briefly with a standard epifluorescence microscope to verify the effectiveness of the staining procedure. In cases in which the staining was weak or incomplete, the bulb was reincubated with the dye solution for an additional $2 \mathrm{~min}$.

Imaging. Neonatal animals whose bulbs had been stained in this way were moved on a detachable stage from the surgical setup to a compound microscope fitted with a Bio-Rad MRC500 laser-scanning confocal system. A standard epifluorescence system (50 W mercury light source; Leitz $\mathrm{H} 2$ excitation/emission cube) was used to view the preparation briefly and to position the stained bulb properly within the field. The confocal system used an argon laser light source ( $488 \mathrm{~nm}$ wavelength). All images were obtained with the laser operating at $10 \mathrm{~mW}$ intensity and a $550 \mathrm{~nm}$ barrier filter; the incident light was further attenuated with a $10 \%$ transmittance neutral density filter. Confocal images were collected through a Leitz $4 \times$ planapo objective (NA 0.12) at a rate of 1 frame/scc; typically between 15 and 20 frames were averaged using Kalman filtration to obtain an optimal image. Because cold anesthesia suppresses both respiration and heart beat, there was no movement artifact during image collection. No more than three images were taken from any animal on a single occasion, and these were stored in computer files for comparison with subsequent images obtained after intervals of $1 \mathrm{hr}$ to 3 weeks. The curvature of the olfactory bulb at its lateral and medial aspects often meant that glomeruli at the periphery of the field 

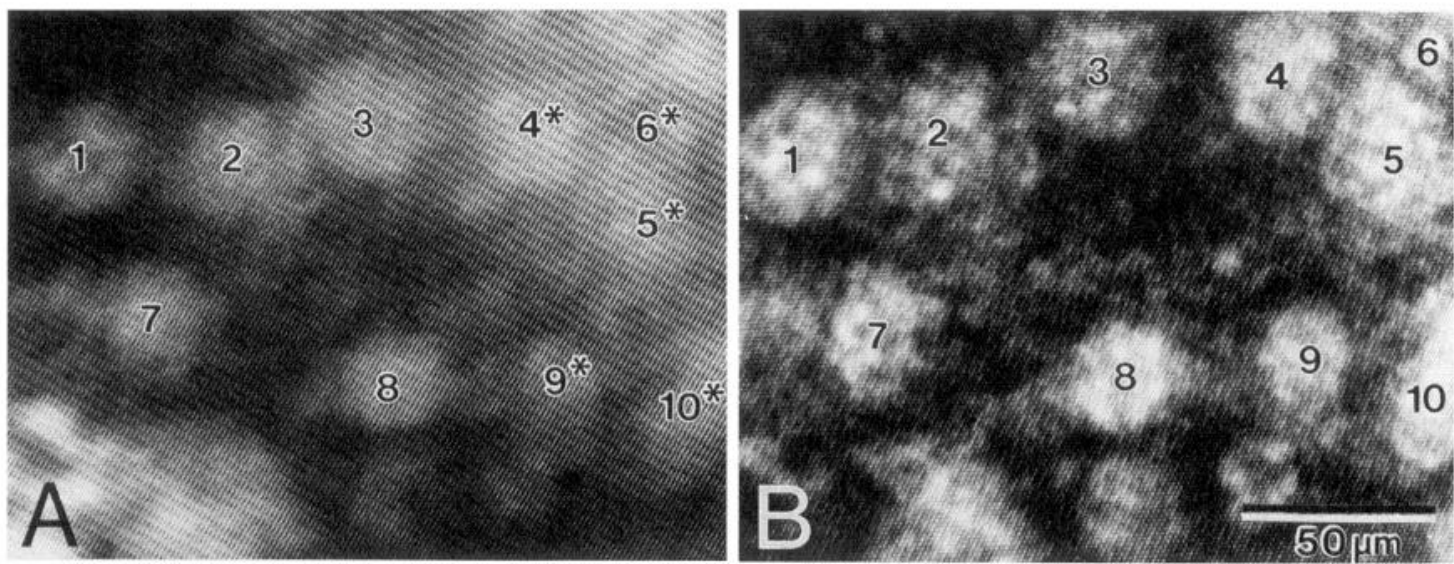

Figure 2. Comparison of images of glomeruli on the dorsal surface of the olfactory bulb in a living 4-d-old mouse obtained with epifluorescence microscopy $(A)$ and laser-scanning confocal microscopy $(B)$. Both images are unprocessed and have been printed with identical contrast. Although the glomerular pattern can be discerned with epifluorescence microscopy, the boundaries of individual glomeruli are seen more clearly with confocal microscopy. The asterisks in $A$ indicate several glomeruli $(4,5,6)$, the borders of which are obscured by out-of-focus information and light scattering arising from fluorescent staining of overlying tissue. The boundaries of these same glomeruli are better seen with the confocal microscope.

were not well seen. Thus, for quantitative comparisons, only the central portions of the images were analyzed.

Images from adult animals were obtained in much the same way as from neonates. Because of the necessarily different anesthesia (cooling is effective only in neonates), adult mice were maintained on a respirator while in the confocal setup. Some modifications were therefore introduced to minimize the movement artifact arising from the pulse and respiration. A small metal arm was fixed to the skull with cyanoacrylate glue to isolate and stabilize the head, and the respirator was turned off briefly while the bulb surface was being scanned. Otherwise, images were collected and stored as described above.

Recovery. After a satisfactory image of the stained bulb had been obtained, infant mice were returned to the setup used for the initial surgery. The wound was rinsed briefly with fresh Ringer's solution, and the edges of the scalp were apposed and cemented in place with surgical glue. The newborn mice were warmed by hand until both heartbeat and respiration were vigorous (3-5 $\mathrm{min})$; they were then allowed to recover for approximately $1 \mathrm{hr}$ in a warm chamber with moist oxygen. When the pups were fully active, they were given an indelible identifying mark and returned to the litter. Most mothers accepted the operated pups without incident; in cases in which a pup was rejected, the remainder of the litter was not used. Among the mothers who did not reject the operated pups (about three out of four), survival rate for the experimental animals was $90 \%$.

Adult mice were returned to the surgical setup and the scalp incision closed in the same manner as for the neonates. When the animal could breath without the aid of the respirator, it was extubated and placed in a warm cage with moist oxygen for $1-2 \mathrm{hr}$ until fully alert. Animals were then transferred to their home cage.

Repeated staining and imaging. Two different methods were used to obtain a second image of the bulb in juvenile and adult animals whose bulbs had been imaged initially in vivo. In some animals a second image was obtained by repeating the in vivo imaging process. Once the bulb was reexposed, the dura was stripped from the surface of the brain. The remaining diffusion barrier presented by the pia/arachnoid was breached with $1.5 \mathrm{M} \mathrm{NaCl}$ for $1 \mathrm{~min}$, and the bulb was stained with RH414 (at $0.1 \times$ the concentration for the initial image) for 30-60 sec. Confocal images were obtained as described above. At the end of the procedure the animal was killed with an overdose of sodium pentobarbital ( 250 $\mathrm{mg} / \mathrm{kg}$ ).

A second method for obtaining a final image of the bulb provided a histological record of the glomerular pattern and was technically easier than reexamining the bulb in vivo. After an appropriate interval, animals whose bulbs had been imaged initially in vivo were administered an overdose of sodium pentobarbital. They were then perfused through the heart with Ringer's solution followed by a solution of $2.5 \%$ glutaraldehyde, $2.5 \%$ paraformaldehyde. The brains were removed and postfixed overnight in the same mixed-aldehyde fixative. The following day, the brains were rinsed with $0.1 \mathrm{~m}$ sodium phosphate buffer and bisected in the midline. Both the previously imaged and the control bulbs were dissected from the remainder of the brain and the glomeruli stained en bloc with the lipophilic dye Sudan black. To visualize glomeruli in this way, the tissue was cleared in propylene glycol for $15 \mathrm{~min}$ and transferred to a solution of 7\% Sudan black B (Sigma Chemical Co., St. Louis, MO) in propylene glycol for an additional $10 \mathrm{~min}$. Next, the blocks were differentiated in a solution of $85 \%$ propylene glycol, $15 \%$ distilled water $(10 \mathrm{~min})$ and subsequently rinsed $(20 \mathrm{~min})$ in distilled water. The stained bulb was then bisected in the horizontal plane, and the dorsal portion of the bulb was mounted on a depression slide in a solution of glycerine $(50 \%)$ and gelatin $(8 \%)$. Stained bulbs were stored at $4^{\circ} \mathrm{C}$ to prevent deterioration.

Controls. To control for possible deleterious effects of the staining and imaging procedures, a series of neonatal and adult mice were prepared for light and electron microscopical examination. Some animals were killed and perfused immediately after imaging, whereas others were examined after intervals of 1-3 weeks. Bulbs for light microscopic analysis using Sudan black were prepared as described above. Bulbs for electron microscopy were obtained from animals that had been perfused through the heart with $0.9 \%$ saline at $37^{\circ} \mathrm{C}$, followed by a solution of $1.25 \%$ paraformaldehyde, $1.25 \%$ glutaraldehyde in sodium phosphate buffer saturated with $\mathrm{CaCl}_{2}$ at $37^{\circ} \mathrm{C}$, and, finally, with $2.5 \%$ paraformaldehyde, $2.5 \%$ glutaraldehyde in the same buffer. The brains were postfixed in aldehyde solution overnight, and further postfixed with $2 \%$ osmium tetroxide. The olfactory bulbs were then removed and embedded in plastic (Epon/Araldite). Both semithin sections for light microscopy and ultrathin sections for electron microscopical analysis were prepared from this material.

\section{Results}

\section{Vital staining characteristics of the olfactory bulb in vivo}

Several dyes of the styryl family can be used to demonstrate the pattern of the glomeruli in the olfactory bulb in living mice (Table 1). Of the dyes examined for relative efficacy, the dicationic dye $\mathrm{N}$-(3-(triethylammonium)propyl)-4(4-(diethylaminophenyl)butadienyl) pyridinium dibromide (RH414; Grinvald et al., 1984) was the most effective. Other dyes that consistently stained living glomeruli included the styryl pyridinium dyes 4-(4-dimethylaminostyryl)- $N$-methylpyridinium iodide (4-di-1ASP) and 4-(4-diethylaminostyryl)- $N$-methylpyridinium iodide (4-di-2-ASP). These two dyes have also been used to stain living motor nerve terminals in muscle (Magrassi et al., 1987) and preganglionic synapses on parasympathetic ganglion cells (Purves et al., 1987). The styryl pyridinium dyes were not, however, as 


\section{Table 1. Efficacy of glomerular staining with various vital fluorescent dyes}

\begin{tabular}{|c|c|c|}
\hline Dye & Quality of staining & Elements stained \\
\hline Auramine-O & - & - \\
\hline Acridine orange 10 -iodecyl bromide & $+1-$ & Nuclei \\
\hline CTM-rhodamine & - & - \\
\hline 4-(4-diethylaminostyryl)- $N$-methylpyridinium iodide (4-di-2-Asp) & +++ & Axons, neuropil \\
\hline 2-(4-dimethylaminostyryl)- $N$-methylpyridinium iodide (2-di-1-Asp) & - & - \\
\hline 4-(4-dimethylaminostyryl)- $N$-methylpyridinium iodide (4-di-1-Asp) & +++ & Axons, neuropil \\
\hline 4-(4-dipropylaminostyryl)- $N$-methylpyridinium iodide (4-di-5-Asp) & ++ & Neuropil, cell bodies \\
\hline 2-(4-dimethylaminostyryl)- $N$-ethylpyridinium iodide (DASPEI) & - & - \\
\hline \multicolumn{3}{|l|}{ 4-(4-dimethylaminophenyl)-butadenyl- $N$-methylpyridinium iodide } \\
\hline (4,4-DMBI) & + & Cell bodies \\
\hline 2-(4-dimethylaminostyryl)- $N$-methylquinolinium iodide (2,4-DMQI) & - & - \\
\hline 4-(4-dimethylaminostyryl)- $N$-methylquinolinium iodide (4,4-DMQI) & $+1-$ & Neuropil \\
\hline 4-(4-hydroxystyryl)- $N$-methylpyridinium iodide (4-HNMI) & $+1-$ & Nuclei \\
\hline$N$-methyl-4-(1-pyrene)vinylpyridinium iodide (NMPVP) & $+1-$ & Nuclei \\
\hline Pyronine-Y & $+/-$ & Nuclei \\
\hline \multicolumn{3}{|l|}{$N$-(3-(triethylammonium)propyl)(4(4-diethylaminophenyl)) } \\
\hline butadienyl) pyridinium dibromide (RH414) & +++ & Neuropil, axons \\
\hline RH415 & $+1-$ & Axons, neuropil \\
\hline RH461 & - & - \\
\hline RH521 & + & Cell bodies, neuropil \\
\hline RH523 & - & - \\
\hline RH687 & - & - \\
\hline RH706 & - & - \\
\hline Rhodamine- $\beta$-isothiocyanate & + & Axons, neuropil \\
\hline Rhodamine 123 & - & - \\
\hline Safranine-O & $+1-$ & Neuropil \\
\hline Sulforhodamine & - & - \\
\hline Texas red & - & - \\
\hline
\end{tabular}

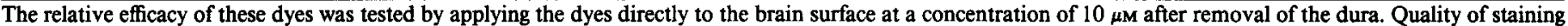

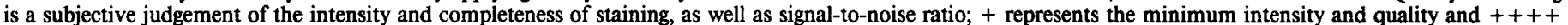
the maximum. A minus sign indicates little or no staining.

effective in penetrating the meninges as the dicationic styryl dye. Accordingly, RH414 was used routinely.

Glomeruli are located well beneath the surface of the bulbs and are covered by both the meninges and the afferent fiber layer (Fig. $1 B$ ). Since both of these overlying structures take up the dyes we used, the glomerular pattern was partially obscured by out-of-focus fluorescence when viewed with a standard epifluorescence microscope (Fig. 2A). Confocal microscopy provided greater resolution of the pattern of olfactory glomeruli by eliminating much of this out-of-focus information and by reducing light scattering from the overlying tissues, even when a $4 \times$ objective was used (Fig. $2 B$ ).

When viewed at somewhat higher magnification $(16 \times$ objective), a punctate distribution of fluorescence was apparent within each glomerulus (Fig. 3A). Neuronal cell bodies and nuclei, however, were not stained. These observations imply that the RH414 stains preferentially some element of the glomerular neuropil. In the PNS, the styryl dyes label primarily nerve terminals (Magrassi et al., 1987; Purves et al., 1987; Balice-Gordon and Lichtman, 1989). To evaluate this possibility in the CNS, we examined a series of $0.5-\mathrm{mm}$-thick brain slices prepared from the olfactory bulbs of saline-perfused animals and stained supravitally with RH414 or 4-di-2-ASP, the two most effective vital dyes. In these slices, both dyes appeared to stain axon fascicles in the primary afferent layer (Fig. $3 B$ ). Single fascicles could often be seen to enter a glomerulus and issue a complex array of branches in a pattern similar to that of primary olfactory afferents demonstrated by routine histological methods (see, e.g., Land et al., 1970).

The relative specificity of the styryl dyes for primary afferent axons was confirmed by examining the in vivo staining pattern after unilateral destruction of the olfactory epithelium. Intranasal $\mathrm{ZnSO}_{4}$ treatment (Margolis et al., 1974) largely eliminated both the axon fascicles and the punctate glomerular pattern normally seen after vital staining (Fig. 4). Therefore, glomerular staining in the intact olfactory system is probably due primarily to the labeling of olfactory afferent axons. The residual staining of glomeruli after chemical deafferentiation, however, suggests that other glomerular elements are stained by styryl dyes. As in the PNS, the mechanism of vital staining with styryl dyes is not understood.

A final issue regarding the adequacy of in vivo staining is the completeness of the glomerular pattern revealed in this way. Consequently, it was necessary to establish that all glomeruli are stained in the living animal and that in vivo and histological methods of visualizing glomeruli are equivalent. We therefore compared the patterns of glomeruli on the dorsal surface of the bulbs stained and imaged in vivo with the glomerular arrangement revealed by immediate en bloc staining with Sudan black, a lipophilic histological stain (Fig. 5). Invariably, there was precise correspondence between the glomerular pattern seen in vivo and that seen histologically. Sudan black, which stains the glo- 
Figure 3. Vital stains are taken up primarily by olfactory afferent axons and terminals. $A$, Higher-power view (16× objective) of individual glomeruli on the lateral aspect of an olfactory bulb vitally stained by topical application of the styryl dye 4-di-2-ASP. A punctate substructure defines each glomerulus. This staining pattern was common to many of the styryl dyes examined ( $\mathrm{Ta}$ ble 1). $B$, Supravital staining of glomeruli with the styryl dye RH414 of a 1 -mm-thick coronal slice from a saline-perfused olfactory bulb $(40 \times$ water-immersion objective). A fascicle of fluorescently labeled axons enters the glomerulus and ramifies extensively within it. The afferent fiber layer, located above the glomeruli, is also stained by the fluorescent dye.
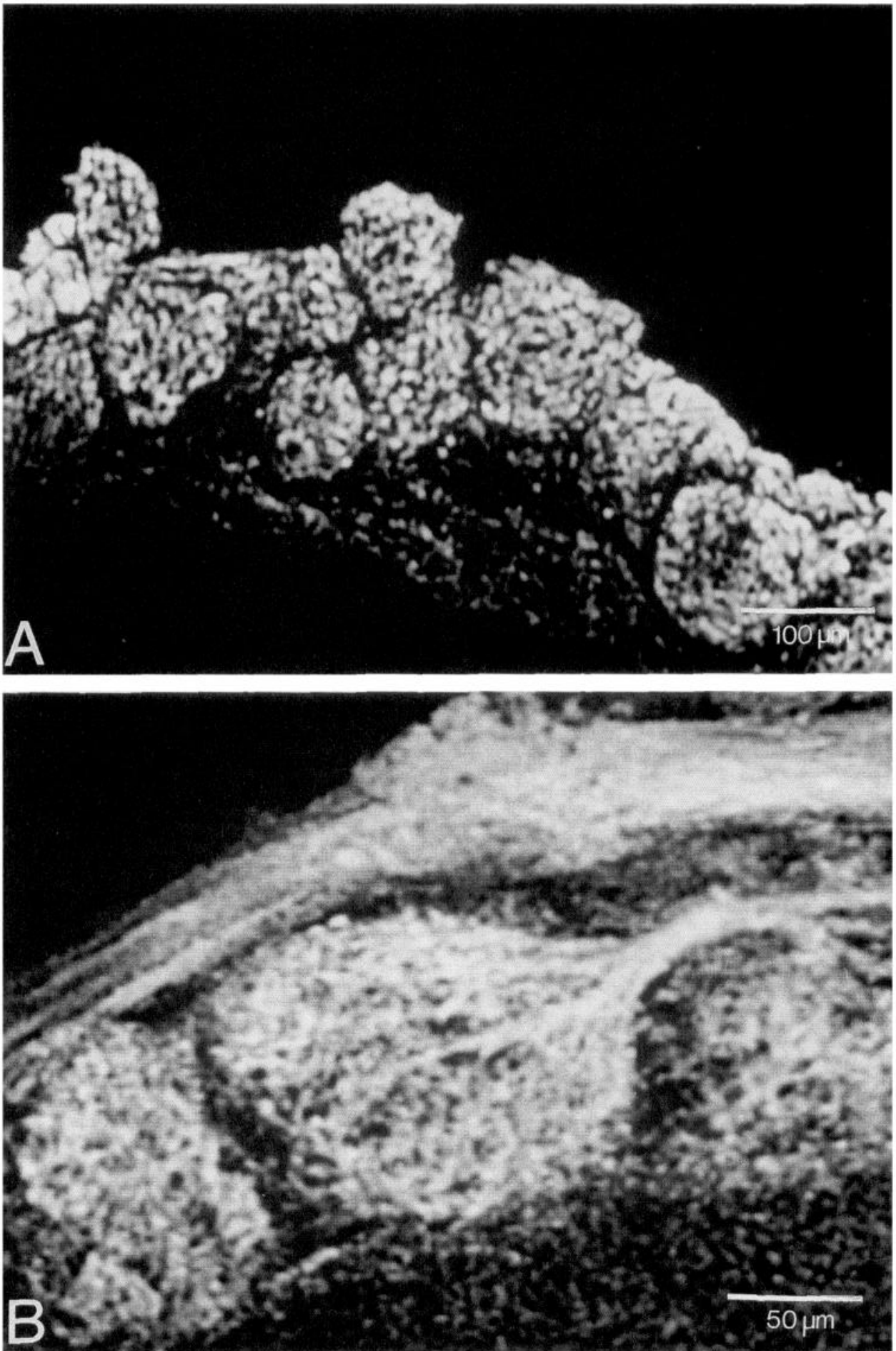

meruli positively and the nuclei of periglomerular cells negatively (see Fig. 5C), showed the spaces between the glomeruli to be filled with tightly packed periglomerular cells (compare Fig. $5 B, C)$.

Control observations of the olfactory bulb after in vivo staining and imaging

To establish that our method of visualizing the bulb in vivo did not compromise its integrity, we carried out further light and electron microscopical analysis of stained and imaged bulbs. Comparisons of Sudan black-stained glomeruli in imaged and contralateral unimaged bulbs of animals after various intervals showed no obvious abnormalities (Fig. 6A). Whether vital staining and imaging leads either to shrinkage or to abnormal growth of glomeruli was evaluated by measuring the size of 300 of these units from imaged bulbs and an equivalent number from the contralateral control bulb (Fig. $6 B$ ). The distribution of glomerular diameters on the treated and untreated sides was sim- 

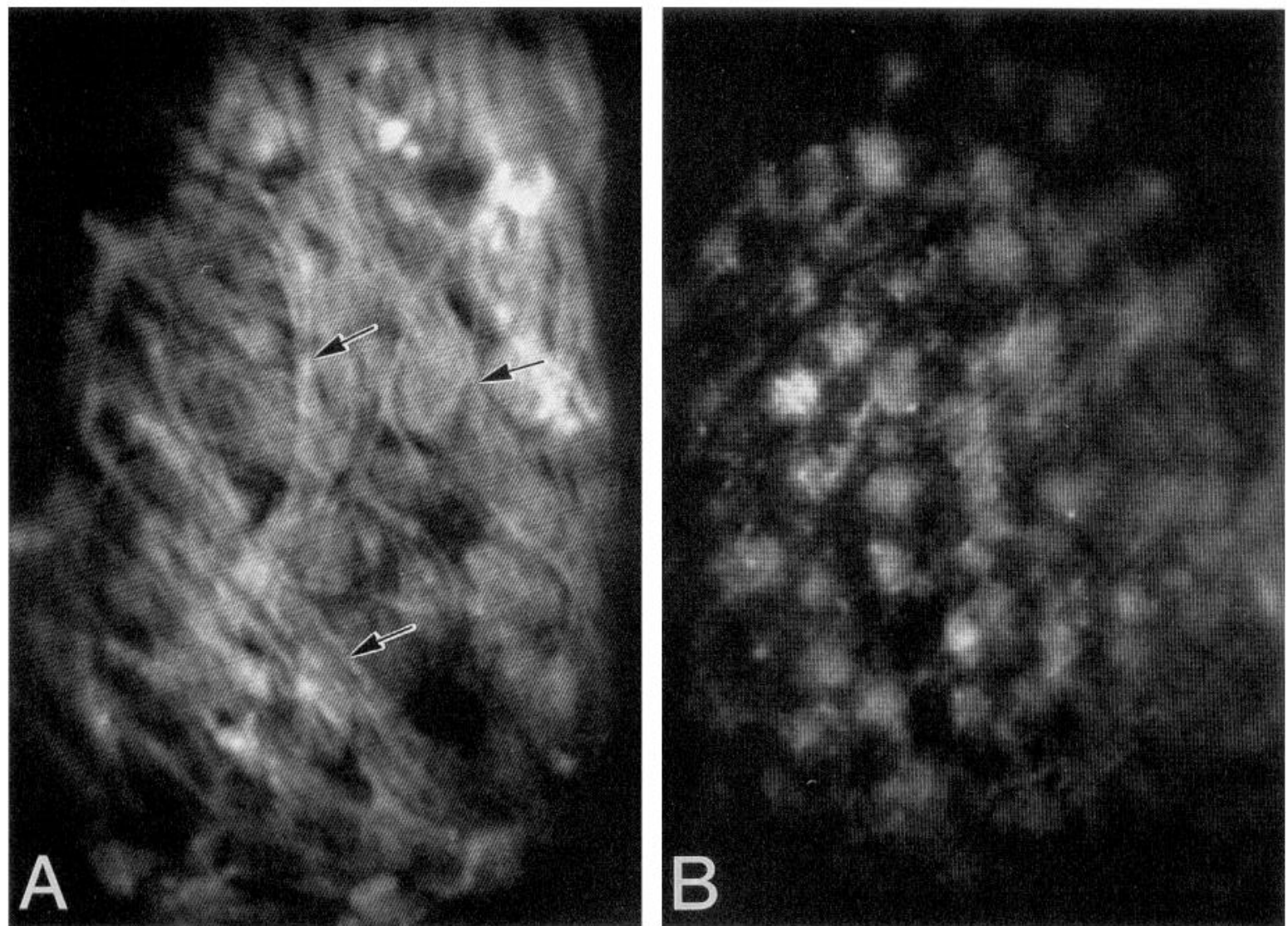

Figure 4. Altered vital staining characteristics of the olfactory bulb after primary afferent fiber ablation. $A$, Low-power view (4 $\times$ objective) of glomeruli on the dorsal surface of the olfactory bulb in a living mouse. $B$, Similar view of the contralateral bulb of the same mouse 12 weeks after unilateral application of the cytotoxin zinc sulfate to the nose. The individual braids of fluorescently stained axons (arrows in $A$ ) and much of the fluorescence arising from terminals within glomeruli on the untreated side $(A)$ are absent after $\mathrm{ZnSO}_{4}$ treatment $(B)$. The persistence of some fluorescence in the absence of the primary afferents probably represents staining of glomerular axons and axon terminals from nonprimary afferents (i.e., periglomerular and short-axon neurons) as well as mitral cell dendrites and the presynaptic terminals of the dendrodendritic contacts that they form. This residual fluorescence indicates that styryl dyes do not stain exclusively primary olfactory axons, but several cellular elements of the glomerulus as well.
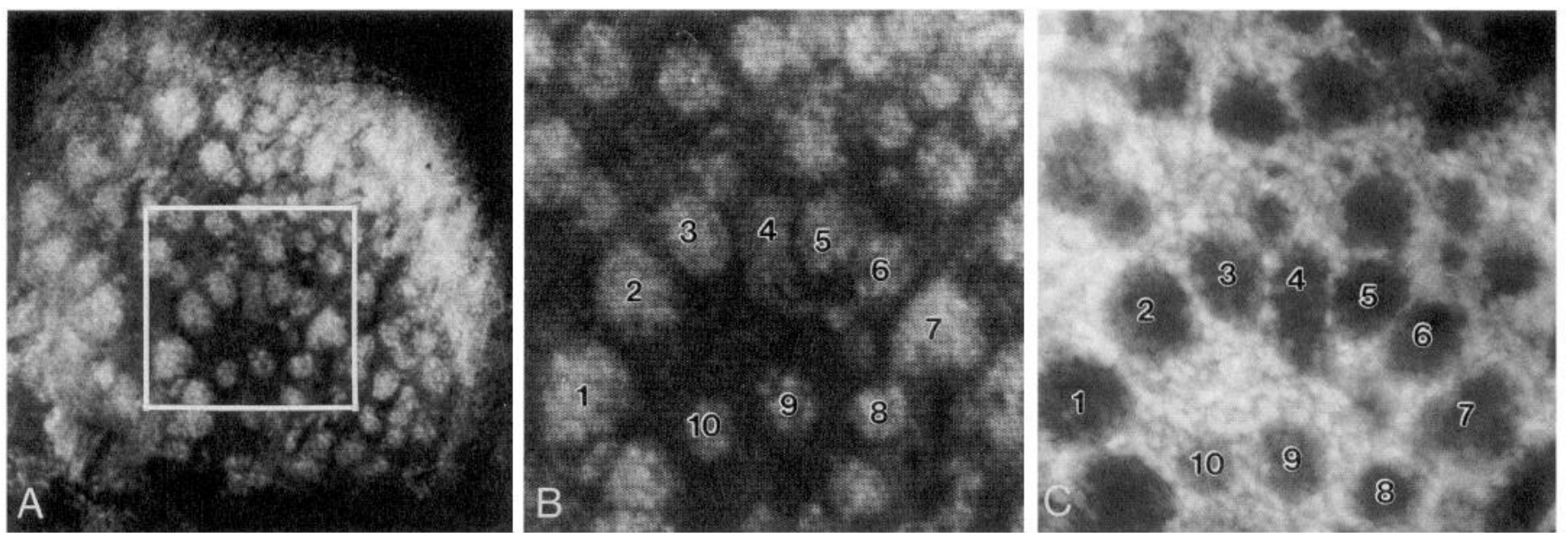

Figure 5. Completeness of glomerular staining in vivo. A, Confocal image of the dorsal surface of the olfactory bulb in a living 4-d-old mouse. B, Higher-magnification confocal image of the glomeruli boxed area in $A$. $C$, The same field after staining with Sudan black and visualization with conventional bright-field microscopy (see Materials and Methods). The bulbs were removed for histological processing immediately after obtaining the images shown in $A$ and $B$. With this method the spaces between glomeruli are seen to be filled with tightly packed, negatively stained periglomerular cells. For reference, 10 glomeruli seen in vivo in $B$ have been labelled. Each of these is seen, in the exact configuration, in the histological preparation shown in $C$. Thus, Sudan black apparently recognizes glomeruli in fixed tissue that correspond exactly with those recognized by vital dyes (see also Table 2). 
Figure 6. Sudan black-stained glomeruli on the dorsal surface of an olfactory bulb after in vivo imaging (left) compared to the unexposed contralateral bulb (right); both images were obtained 2 weeks after the initial in vivo staining and confocal imaging. Histograms of the distribution of the diameters of a total of 300 glomeruli measured in this and another pair of bulbs. The distribution and the means of glomerular size are similar in the imaged bulbs and the unexposed control bulbs.

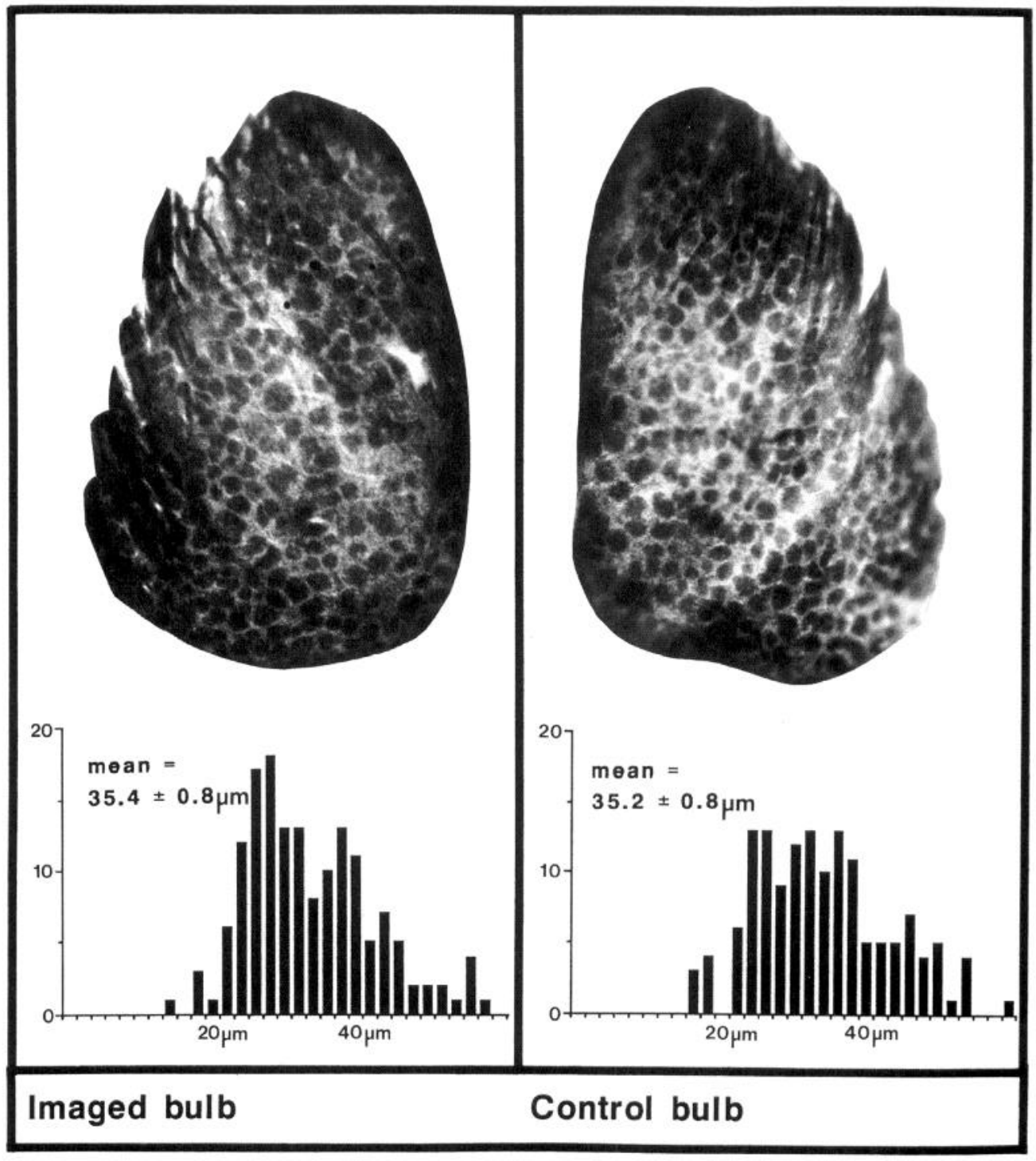

ilar. The mean value measured in Sudan black preparations ( 35 $\mu \mathrm{m})$ is in close agreement with the glomerular diameter measured in histological sections from normal animals of similar age (Pomeroy et al., 1990).

We did not see any differences between $1 \mu \mathrm{m}$ sections through the dorsal surface of olfactory bulbs fixed immediately after imaging and sections from the contralateral, unstained bulb of the same animal (Fig. 7). The pial surface appeared normal, the nerve fiber layer was intact, the underlying glomerular neuropil and the periglomerular spaces were normally arranged, and the external plexiform and mitral cell layers were identical to those in the control sections. Similar results were obtained when a 2 week interval was allowed to elapse between the initial staining and imaging and the control observations (not shown).

The dorsal surface of olfactory bulbs fixed acutely, or following 2 week postimaging intervals, was also indistinguishable from the contralateral control at the ultrastructural level (Fig. 8 ). The small-caliber unmyelinated axons of the olfactory nerve fiber layer were intact, the adjacent glial cells were of usual size and appearance, and the synaptic organization of the underlying glomerular neuropil was similar to that on the control side. Both the primary afferent synaptic terminals and other presynaptic processes arising from local circuit neurons within the bulb were normal in appearance, as were the dendritic profiles of mitral and tufted cells within the glomeruli.

\section{The addition of glomeruli to the developing olfactory bulb observed in vivo}

Having assured ourselves about the adequacy of the technique, we first applied it to an examination of the developing olfactory bulbs. Although counts of glomeruli in serial sections have been taken to imply that new circuits are constructed for a prolonged postnatal period in the mouse (e.g., Pomeroy et al., 1990), other interpretations are possible (e.g., Meisami, 1990). Moreover, it is not known in any system whether the complex circuits initially formed in the brain are stable or whether some are lost during development. To address these issues, we observed the pattern of glomeruli on the dorsal surface of the bulbs of neonatal (45-d old) mice and then allowed them to recover (see Materials and Methods). A second observation of the same bulbs was made 1-3 weeks later. Representative examples of such images over different intervals are shown in Figures 9-11. 

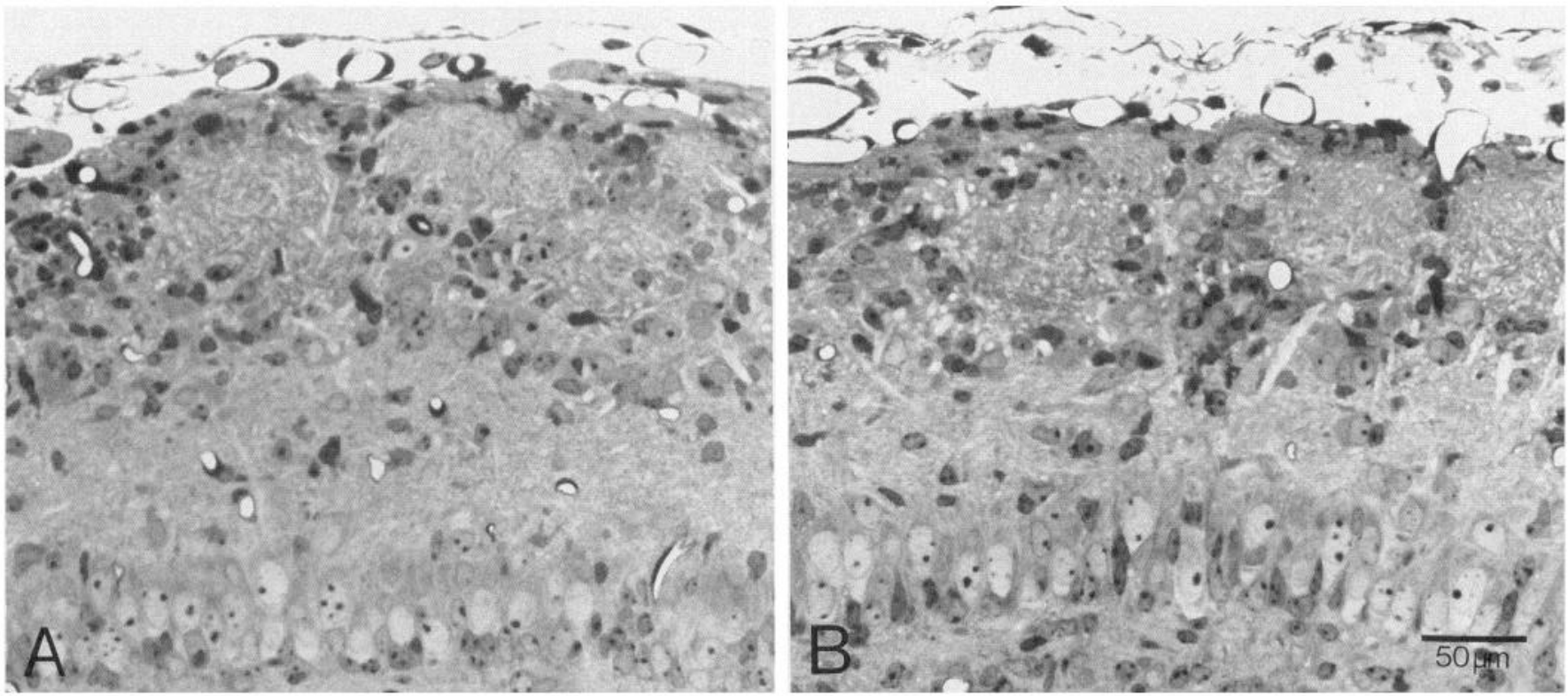

Figure 7. Integrity of imaged bulb observed in light microscopical sections. $A$, Micrograph of 1- $\mu$ m-thick coronal section through the dorsal surface of the olfactory bulb in a 4-d-old mouse after in vivo staining and imaging. $B$, Similar section from the contralateral unexposed bulb. This animal was perfused about $1 \mathrm{hr}$ after the staining and imaging procedure was completed. There is no sign of acute damage in the pial covering of the bulb, which is slightly elevated on both sides, probably from the histological processing. The glomerular neuropil, external plexiform layer, and mitral cell layer appear the same on the imaged and unexposed side. Light microscopical images obtained after waiting up to 3 weeks after staining and imaging were similar.
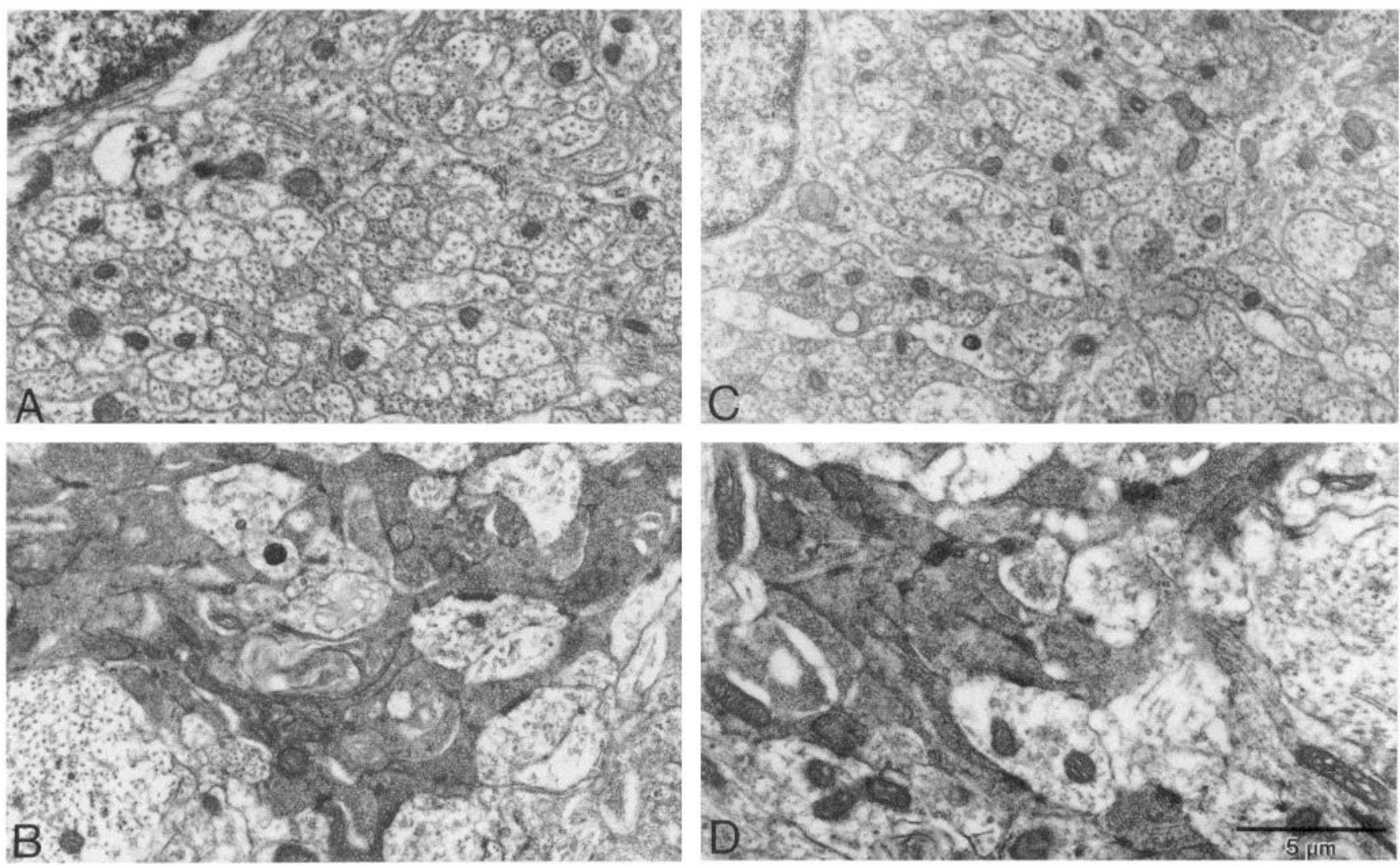

Figure 8. Integrity of imaged bulbs observed at the electron microscopical level: electron micrographs of the olfactory nerve fiber layer $(A)$ and the glomerular neuropil $(B)$ from the dorsal surface of an olfactory bulb prepared 2 weeks after in vivo staining and imaging. Comparable regions from the dorsal surface of the contralateral unexposed bulb are shown in $C$ and $D$. The small, delicate olfactory afferent axons are intact and the glial cells that ensheath them appear normal $(A, C)$. Dark, vesicle-rich afferent terminals that characterize the glomerular neuropil are seen making synapses on a variety of postsynaptic processes $(B, D)$. 


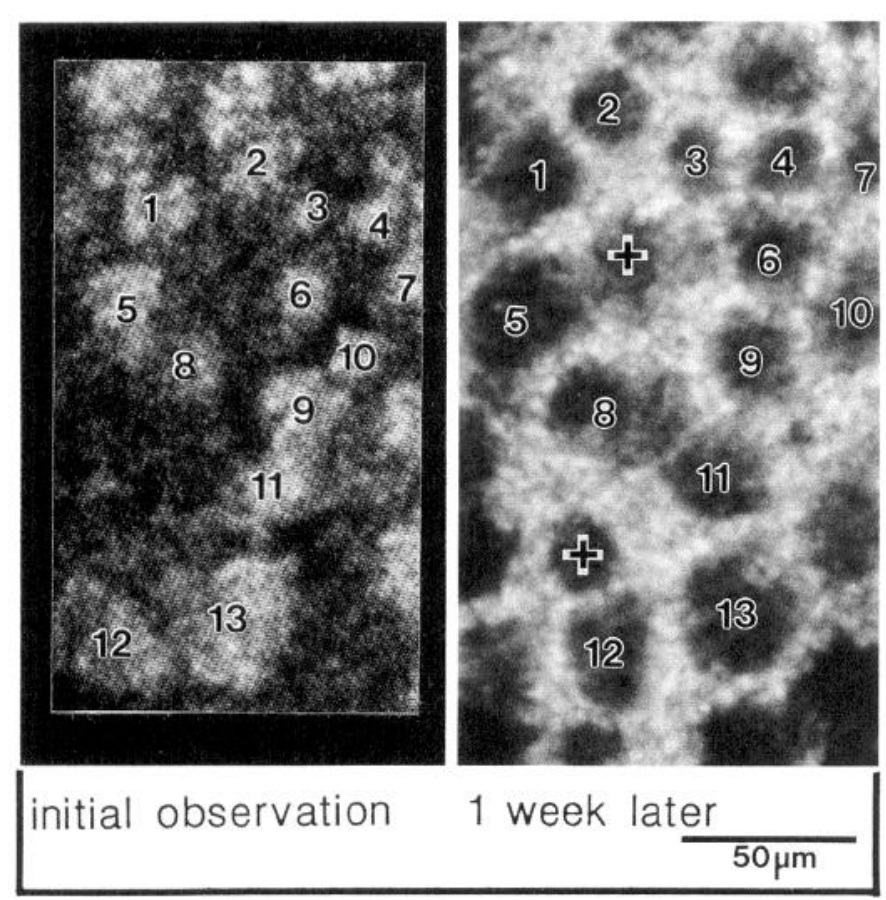

Figure 9. Example of glomerular pattern observed over a 1 week interval. The left panel shows initial in vivo image; the final image 1 week later (right panel) was obtained by Sudan black staining. Numbers indicate corresponding glomeruli; new glomeruli are indicated by plus signs. The two glomeruli that have appeared during the 1 week interval are somewhat smaller and have formed in the spaces between existing glomeruli. Note that most of the glomeruli initially present have increased appreciably in size.

Repeated observations of the same region of the olfactory bulbs in developing animals showed that glomeruli are added gradually and progressively during the first weeks of postnatal life. A quantitative summary of these results from 28 mice is presented in Table 2 . The percentage increase of new glomeruli seen in different animals-between $5 \%$ and $30 \%$ over a 1 week interval and between $10 \%$ and $70 \%$ over a $2-3$ week intervalis in general agreement with estimates of the range of glomerular addition made using standard histological techniques (Pomeroy et al., 1990). No deletion of any of the 882 identified glomeruli present initially was observed over intervals of 1-3 weeks.

Identified glomeruli observed over time also enlarge progressively (see Figs. 9-11). The magnitude of this enlargement observed in vivo is similar to estimates of glomerular growth based upon measurements in Nissl-stained histological sections through the developing mouse olfactory bulb at different ages (Pomeroy et al., 1990). The mean increase in the cross-sectional area of glomeruli measured in vivo was $141 \%$ over 1 week $(n=53$ pairs of homologous glomeruli) and $214 \%$ over 3 weeks ( $n=56$ pairs of homologous glomeruli). The rate of growth in fixed specimens from normal animals was approximately $100 \%$ for 1 week intervals and $180 \%$ for 3 week intervals (see Pomeroy et al., 1990). The absolute size of glomeruli in the Nissl-stained sections at all ages was somewhat smaller than the corresponding in vivo values, presumably due to tissue shrinkage during histological preparation.

\section{The pattern of glomeruli in adult animals observed in vivo}

Based on histological observations, the number of glomeruli in the mouse olfactory bulbs gradually approaches adult values over the first 3 months of postnatal life (Pomeroy et al., 1990). We wished to determine whether the stability of glomerular number in maturity represents an underlying stability of individual glomeruli. In contrast to observations in developing animals, we saw no addition of glomeruli over similar intervals ( 2 weeks) in fully adult mice (Fig. 12). In addition to facilitating comparison with the observations over similar times in developing animals, a 2 week interval represents approximately half the estimated turnover time of the olfactory receptor cells in mice (Graziadei and Monti-Graziadei, 1979; but see Hinds et al., 1984). No additions or deletions from the initial population (161 glomeruli in 11 adult animals) were observed over this period, nor was there any consistent change in glomerular size-

Table 2. Summary of glomerular addition in developing olfactory bulbs viewed after various intervals

\begin{tabular}{|c|c|c|c|c|c|c|c|c|c|c|c|c|c|c|c|}
\hline \multicolumn{4}{|c|}{0 time interval } & \multicolumn{4}{|c|}{1 week interval } & \multicolumn{4}{|c|}{2 week interval } & \multicolumn{4}{|c|}{3 week interval } \\
\hline$n_{0}$ & $n_{f}$ & $g l_{+}$ & $g l_{-}$ & $n_{0}$ & $n_{f}$ & $g l_{+}$ & $g l_{-}$ & $n_{0}$ & $n_{f}$ & $g l_{+}$ & $g l_{-}$ & $n_{0}$ & $n_{f}$ & $g l_{+}$ & $g l_{-}$ \\
\hline 50 & 50 & 0 & 0 & 48 & 54 & 6 & 0 & 63 & 74 & 11 & 0 & 19 & 26 & 7 & 0 \\
\hline 41 & 41 & 0 & 0 & 44 & 49 & 5 & 0 & 42 & 49 & 7 & 0 & 21 & 25 & 4 & 0 \\
\hline 42 & 42 & 0 & 0 & 41 & 44 & 3 & 0 & 30 & 37 & 7 & 0 & 20 & 26 & 6 & 0 \\
\hline 53 & 53 & 0 & 0 & 24 & 30 & 6 & 0 & 54 & 65 & 12 & 0 & 20 & 25 & 5 & 0 \\
\hline 81 & 81 & 0 & 0 & 33 & 36 & 3 & 0 & 43 & 51 & 8 & 0 & 16 & 19 & 3 & 0 \\
\hline 52 & 52 & 0 & 0 & 27 & 33 & 6 & 0 & 29 & 35 & 6 & 0 & 24 & 34 & 10 & 0 \\
\hline 53 & 53 & 0 & 0 & 25 & 31 & 6 & 0 & 30 & 39 & 9 & 0 & 38 & 43 & 5 & 0 \\
\hline 51 & 51 & 0 & 0 & 23 & 25 & 2 & 0 & 29 & 41 & 12 & 0 & & & & \\
\hline 51 & 51 & 0 & 0 & 38 & 46 & 8 & 0 & 20 & 29 & 9 & 0 & & & & \\
\hline 41 & 41 & 0 & 0 & 30 & 35 & 5 & 0 & 14 & 16 & 2 & 0 & & & & \\
\hline 48 & 48 & 0 & 0 & & & & & 37 & 44 & 7 & 0 & & & & \\
\hline 39 & 39 & 0 & 0 & & & & & & & & & & & & \\
\hline 25 & 25 & 0 & 0 & & & & & & & & & & & & \\
\hline 627 & 627 & 0 & 0 & 333 & 383 & 50 & 0 & 391 & 481 & 90 & 0 & 158 & 208 & 50 & 0 \\
\hline adde & & & & adde & & & & adde & & & & adde & & & \\
\hline
\end{tabular}

$n_{0}$, number of identified glomeruli observed initially; $n_{f}$ final number of glomeruli observed in same region after the interval indicated; $g l_{+}$, number of new glomeruli added; $g l_{-}$, number of glomeruli lost. 


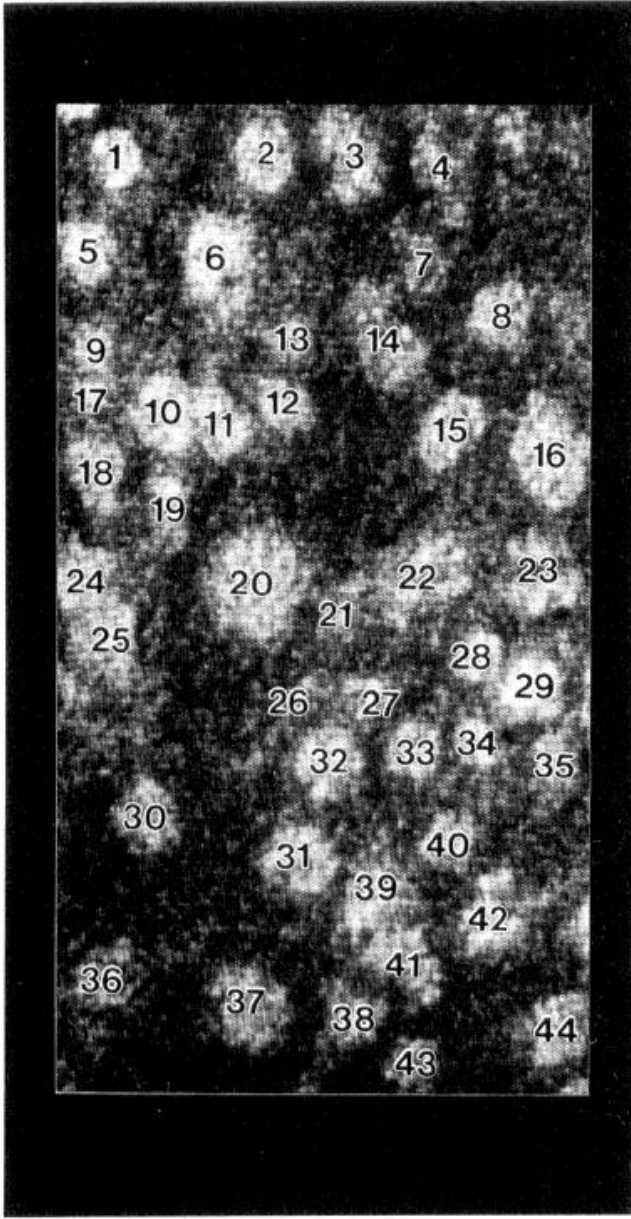

initial observation

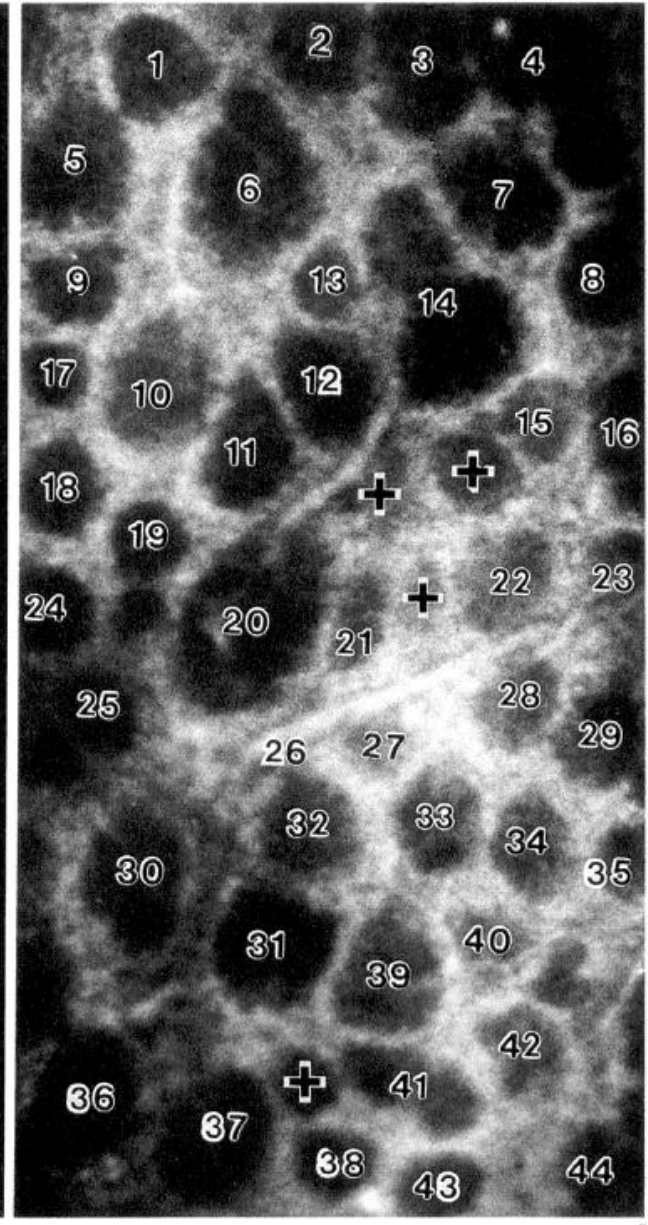

2 weeks later

$50 \mu \mathrm{m}$

Figure 10. Example of glomerular pattern observed over a 2 week interval; designations are as in Figure 9. Four new glomeruli have been added to the cohort of glomeruli present initially; none of the original glomeruli have been lost. measurements of 51 pairs of homologous glomeruli showed no significant change in the mean diameter or cross-sectional area of these units over the 2 week interval. In contrast, the size of identified glomeruli observed in vivo increased by $214 \%$ during the first several weeks of postnatal life.

\section{Discussion}

At present, vital fluorescent staining and repeated imaging of the brain provide the only definitive means of assessing the dynamic behavior of complex brain circuits. Our results show that one class of such circuits, olfactory glomeruli, can be observed in living animals without compromising their integrity. We have drawn three conclusions from the observation of identified glomeruli over time in developing and adult mice. First, glomeruli are added progressively to the developing olfactory bulb. Second, glomerular addition proceeds by the intercalation of new units among a stable set of preexisting circuits. Third, the overall pattern of glomeruli in fully mature animals is stable, at least over the intervals examined here. This latter point is also supported by the average constancy of glomerular numbers in adult mice over an interval of 6 months (Pomeroy et al., 1990).

The physiological integrity of the tissue observed is a central concern in evaluating the utility of any vital imaging strategy.
The surgical procedure, staining, and imaging techniques might create artifactual change (or stability) that would confound the interpretation of dynamic processes. Several observations support the physiological nature of the results we describe in the developing and adult olfactory bulb. We detected no histological or ultrastructural damage in tissue that had been stained and imaged, either acutely or over intervals of several weeks. Thus, a laser-scanning confocal microscope-with its greater ability to define structures such as glomeruli-can be used to image nervous tissue in the intact animal. Further, the results obtained using these vital staining and imaging techniques are themselves an internal control: under essentially identical conditions, changes in glomerular number, size, and pattern are observed in developing animals but not in adults studied over similar intervals. Finally, a histological stain, Sudan black, which demonstrates both glomeruli and the cell-dense periglomerular spaces, confirms that the in vivo images include all the glomeruli present. The agreement between in vivo and histological images of the same specimen argues against the existence of cryptic glomeruli that escape detection (Meisami, 1991).

The process of glomerular addition observed in living mice during the first weeks of life is consistent with that inferred from quantitative assessment of fixed specimens (Meisami, 1989; Pomeroy et al., 1990). Observations of olfactory bulb devel- 
Figure 11. Example of glomerular pattern observed over a 3 week interval; designations are as in Figure 9. Two new glomeruli have been added to the stable cohort of 36 glomeruli present initially.

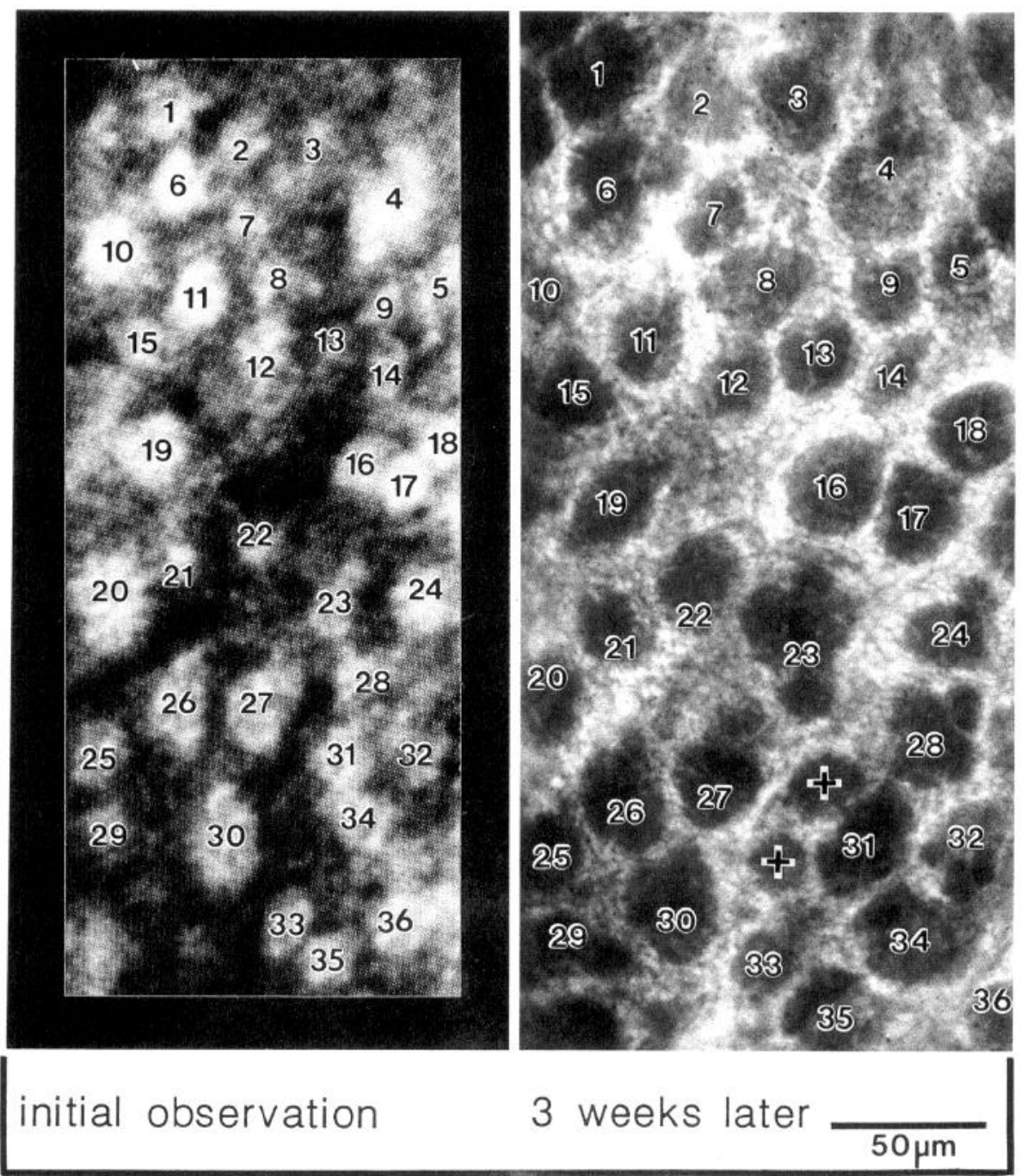

factory bulb also provide an occasion to reconsider the merits of a popular hypothesis about the development and maintenance of patterned connections in the CNS. Many neurobiologists believe that the formation of adult circuitry in the mammalian brain proceeds by the elimination of some circuits from an initial excess (Changeux et al., 1973; Young, 1973, 1979; Changeux and Danchin, 1976; Edelman, 1987). The progressive addition of glomeruli to the mouse olfactory bulb shows that, at least in this part of the rodent brain, neural development proceeds by the continued addition and growth of neural elements, and the ongoing organization of these elements into new, complex circuits. The loss of complex circuitry appears to play little or no part in establishing the final number and arrangement of these units.

The mode of glomerular addition observed in living animals also casts new light on the question of how iterated circuitry throughout the brain is formed during development. The addition of new afferents to the bulb from an expanding olfactory epithelium (Meisami, 1989; Pomeroy et al., 1990) presumably provides an increasing supply of axon terminals to instigate the formation of new glomeruli. Although the addition of entirely new axons postnatally is an unusual (but not unique) feature of the developing olfactory system (see, e.g., Nurse and Diamond, 


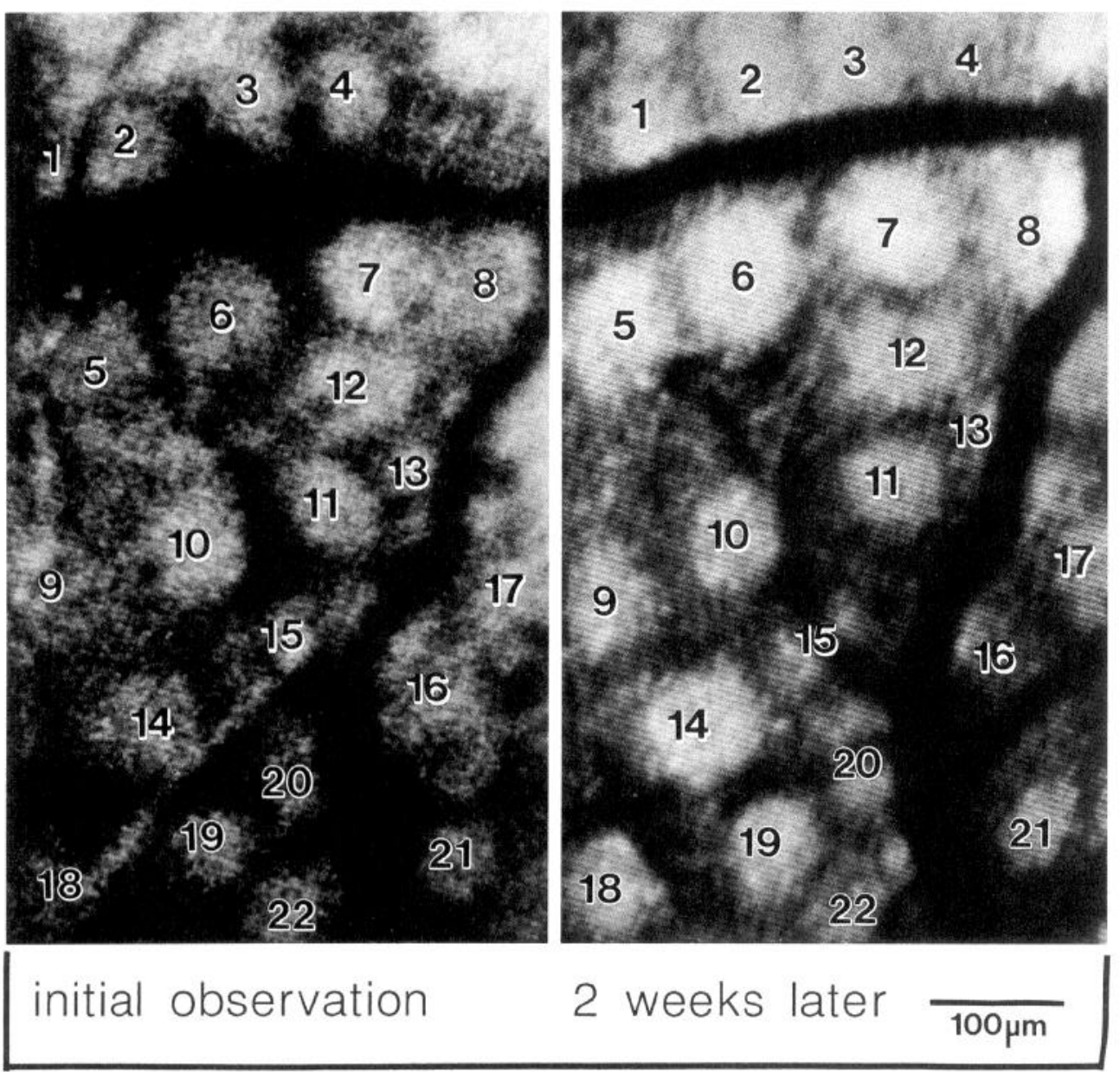

Figure 12. Example of glomerular pattern in a mature mouse observed over a 2 week interval; designations are as in Figure 9. In this case, however, the final image was obtained by repeated vital staining rather than by Sudan black histology. In the adult mouse, large blood vessels not present in neonates also provide lancinarks for comparison. All of the 20 glomeruli indicated in the initial image were visible in the second image; no new glomeruli are apparent over this interval.
1984; Young, 1985; Turner and Cepko, 1987; Mills et al., 1989), the growth of terminal arborizations from immature axons is presumably universal in the developing brain. Since a distinction between terminal branches arising from new axons and additional branches from existing axons is probably not made by target neurons, and since dendritic growth and elaboration occur throughout the developing brain, the development of glomeruli may be qualitatively (if not quantitatively) representative of circuitry elsewhere in the telencephalon. The local growth of neurites could account for the emergence of other modular circuits generally thought to be formed by selective elimination of axons or dendrites from a diffuse initial pattern. In principle, the approach we have described here could be used to determine whether ongoing circuit addition occurs in other regions of the growing brain.

\section{References}

Astic L, Saucier D (1983) Ontogenesis of the functional activity of guinea-pig olfactory bulb: autoradiographic study with the 2-deoxyglucose method. Dev Brain Res 10:257-263.

Balice-Gordon RJ, Lichtman JW (1989) Competing motor nerve terminals and the acetylcholine receptors underlying them are rearranged during synapse elimination. Soc Neurosci Abstr 15:165.

Changeux J-P, Danchin A (1976) Selective stabilisation of developing synapses as a mechanism for the specification of neuronal networks. Nature 264:705-712.

Changeux J-P, Courrège P, Danchin A (1973) A theory of the epigenesis of neuronal networks by selective stabilization of synapses. Proc Nat Acad Sci USA 70:2974-2978.
Edelman GM (1987) Neural Darwinism: the theory of neuronal group selection. New York: Basic.

Farbman AI, Margolis FL (1980) Olfactory marker protein during ontogeny: immunohistochemical localization. Dev Biol 74:205-215.

Fenstermacher JD, Rappoport SI (1984) Blood-brain barrier. In: Handbook of physiology. The cardiovascular system IV, Part 2 (Renkin EM, Michel CC, eds), pp 969-1000. Bethesda, MD: American Physiological Society.

Fine A, Amos WB, Durbin RM, McNaughton PA (1988) Confocal microscopy: applications in neurobiology. Trends Neurosci 11:346351 .

Friedman B, Price JL (1984) Fiber systems in the olfactory bulb and cortex: a study in adult and developing rats, using the Timm method with the light and electron microscope. J Comp Neurol 233:88-109.

Golgi C (1874) Sulla fina struttura dei bulbi olfattorii. Riv Sper Freniatr Med Leg Alienazioni Ment 1:405.

Graziadei PPC, Monti-Graziadei GA (1979) Neurogenesis and neuron regeneration in the olfactory system of mammals. II. Degeneration and reconstitution of the olfactory sensory neurons after axotomy. J Neurocytol 8:197-213.

Green, CJ (1979) Animal anesthesia. London: Laboratory Animals.

Grinvald SA, Anglister L, Freeman JA, Hildesheim R, Manker A (1984) Real time optical imaging of naturally evoked electrical activity in intact frog brain. Nature 308:848-850.

Hinds JW, Hinds PL (1976) Synapse formation in the mouse olfactory bulb. J Comp Neurol 169:15-40.

Hinds JW, Hinds PL, McNelly NA (1984) An autoradiographic study of the mouse olfactory epithelium: evidence for long-lived receptors. Anat Rec 210:375-383.

Hubel DH (1988) Eye, brain, and vision. New York: Scientific American.

Inoué S (1986) Video microscopy. New York: Plenum.

LaMantia A-S, Purves D (1989) Development of glomerular pattern visualized in the olfactory bulbs of living mice. Nature 341:646-649. 
Land LS, Eager RP, Shepherd GM (1970) Olfactory nerve projections to the olfactory bulb in rabbit: demonstration by means of a simple ammoniacal silver degeneration method. Brain Res 25:250-254.

Magrassi L, Purves D, Lichtman JW (1987) Fluorescent probes that stain living nerve terminals. J Neurosci 7:1207-1214.

Margolis FL, Roberts N, Ferriero D, Feldman J (1974) Denervation in the primary olfactory pathway of mice: biochemical and morphological effects. Brain Res 81:469-483.

Meisami E (1989) A proposed relationship between increases in the number of olfactory receptor neurons, convergence ratio and sensitivity in the developing rat. Dev Brain Res 46:9-19.

Meisami E (1990) Two views on formation of olfactory glomeruli based on numerical study of olfactory neurons, glomerular and mitral cells in rat, hamster and rabbit. Soc Neurosci Abstr 16:630.

Meisami E (1991) Evidence for the "early formation" of glomeruli in the developing mammalian olfactory bulb. Chem Senses 16:554.

Mills LR, Nurse CA, Diamond J (1989) The neural dependency of Merkel cell development in the rat: the touch domes and foot pads contrasted. Dev Biol 136:61-74.

Mountcastle VB (1978) An organizing principle for cerebral function: the unit module and the distributed system. In: The mindful brain: cortical organization and the group-selective theory of higher brain function (Edelman GM, Mountcastle VB, eds), pp 7-50. Cambridge, MA: MIT Press.

Nurse CA, Diamond J (1984) A fluorescent microscopic study of the development of rat touch domes and their Merkel cells. Neuroscience 11:509-520.

Pomeroy SL, LaMantia A-S, Purves D (1990) Postnatal construction of neural circuitry in mouse olfactory bulb. J Neurosci 10:1952-1966.
Purves D, Voyvodic J (1987) Imaging mammalian nerve cells and their connections over time in living animals. Trends Neurosci 10 398-404.

Purves D, Hadley RD, Voyvodic JT (1986) Dynamic changes in the dendritic geometry of individual neurons visualized over periods of up to three months in the superior cervical ganglion of living mice. J Neurosci 6:1051-1060.

Purves D, Voyvodic JT, Magrassi L, Yawo H (1987) Visualization of synapses over time in living animals. Science 238:1122-1126.

Rakic P (1988) Specification of cerebral cortical areas. Science 241: 170-176.

Scott JW, Harrison TA (1987) The olfactory bulb: anatomy and physiology. In: Neurobiology of taste and smell (Finger TE, Silver WL, eds), pp 151-178. New York: Wiley.

Shepherd GM (1972) Synaptic organization of the mammalian olfactory bulb. Physiol Rev 52:864-917.

Turner DL, Cepko CL (1987) A common progenitor for neurons and glia persists in rat retina late in development. Nature 328:131-136.

White JG, Amos WB, Fordham M (1987) An evaluation of confocal versus conventional imaging of biological structures by fluorescence light microscopy. J Cell Biol 105:41-48.

Young JZ (1973) Memory as a selective process. In: Australian Academy of Science report: symposium on biological memory, pp 25-45. Canberra: Australian Academy of Science.

Young JZ (1979) Learning as a process of selection and amplification. J R Soc Med 72:810-814.

Young RW (1985) Cell differentiation in the retina of the mouse. Anat Rec 212:199-205. 